\title{
Collaborative and statutory pathology practice
}

\section{Commentary}

The territory of legal habitude and law amendments is affiliated to the delivery of healthcare, both at the macro and the micro health levels. The legal foundations are a dynamic and a promptly up surging field with social, scientific, fiscal, philosophical and political consequences. All aspects of medicine and healthcare are affected by and are required to have awareness of eventualities of the law. The healthcare remodelling presumably will organize and finance the healthcare development. All forms and manner of litigation are on the rise, medical devices, blameworthy deaths, confidential patient and health information on the internet, advances in science and technology, genetic and reproductive research, the evolving conventionalities, newer frames of reference etc.

The legal framework is influenced by the ethical and moral issues, which may be the rules versus virtual insights, delineating and exercising facts and principles. The comprehension of infractions and misconduct, the concept of negligence, the fundamentals of standard care and the role of corroborators required for assessing risk in daily pathology practice, comprehending the litigation process and the knack to handle high risk specimens/diagnosis/depositions are of practical value in a good medical practice. Medico legal issues for Histopathologist are different from their Clinical counterparts due to minimal patient contact. Following appropriate ground rules and circumspection while deputing work to technicians, non-pathological staff are essential.Glitches in diagnosis and documentation are the most common dilemmas. Deprivation of adequate clinical data, expertise of the laboratory staff, clinicopathologic discussions, cooperation in quality control programmes, audit and constant standard operating procedures are needed to disclose and abbreviate errors and avert atrocious outcomes to healthcare recipients. Wet, formalin-fixed tissue should be kept for as long as possible, along with chronicled reports, paraffin blocks for a lifetime and histopathological slides for a minimum period of ten years. Processed tissue can be used for research and education and exhibited in the museum. Authorization for surgery and autopsy can be amended to include storage and display of human organs for education and research. Good medical practice in Histopathology includes consulting with colleagues, apprising the treating clinician of the surgical resection being referred to another direction for a second opinion. With the advent of ancillary techniques, the medico legal arena and diagnosis protocol is likely to diversify (Figure 1).

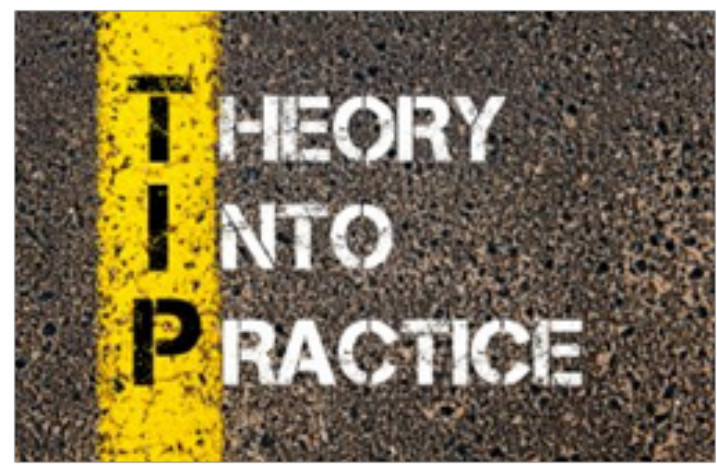

Figure I Good medical practice in Histopathology.

\author{
Volume 6 Issue I - 2018
}

Anubha Bajaj
University Panjab University, India

Correspondence: Anubha Bajaj, Designation Consultant Pathologist, University Panjab University, Chandigarh, India, Tel 00-9I-II25II7399, 009198II693956,

Email anubha.bajaj@gmail.com, anubha.bajaj@yahoo.com

Received: November 10, 2017 | Published: January 08, 2018

Evidence based medicine describes the classic science that accomplishes, establishes the conclusion and incorporates in the law, identification and classification of the erratum, the affiliation of negligence, standard of care and fallacy, harmony of the patient rights with the medical and scientific facts.

\section{Pertinence and conventions}

Erroneous diagnosis is a considerable and an expensive division of medical erratum's. Incorrect diagnosis induces deferred and unsuitable treatment. So the relevant inquiries are the motive and the manner the calamity has arisen and to allocate and account for the legal repercussions. The legal channel commences with the identification, description and manifestation of the blunder. A clear, logical exposition of error exemplifies the particular conclusions which are omitted, fallacious or postponed. The sequel indicates the error. The inquisite clarification is rationally and consecutively adopted in pre analytical, analytical and post analytical succession. An etiquette of national pathology association is preferable for access by the experts/legal cabinet. Pre analytical determinants are the purpose of testing, the sampling apropos, and the requisite clinical dossier. Analytical agents are coordination and technical methodologies in the laboratory, concerned with sampling, tissue processing, slide preparation, appurtenant employment of adjuvant techniques. Post analytical aspects include specific outcomes, ultimate communiqués, clinically channelized and transported a communication which should be exhaustive and accurate. Computerized data necessitates a manner of authentication and approval, a composite report and befitting distribution. Intellective inaccuracies, partialities and interrogations are frequent determinants of diagnostic misconjecture.

Expert pathologists are requested to check the slides thoroughly. Camouflaged reassessment is essential for impartial appraisal and in non legal circumstances. On reconsideration, defective morphological interpretations and the category of examination can control the causatum. Review for legal audit ensues several years following the primordial scrutiny. A judicial process that should be consistently capable of arriving at decisions guided by an evidence- base comprising of complex scientific dossier including pathologic assimilation of tissue specimens. The legal conformity needs to contemplate the science underlying the assertions of medical expertise, medical oversight and malfeasance. Encompassing the practice of science based medical evidence and having cardinal elements of concise 
assessments should be incorporated in a legal ability in medicine and healthcare. ${ }^{1}$

Tort law has two appended missions, rectification of adversity and restraint from misjudgement. When a pathologist is held accountable, it admonishes the compatriots to counteract a reoccurrence of the disaster. Partial appraisals and consequences accounts for the inhibition enterprise to go awry. Legal aspects in Virtual Pathology: The conjectures of the medical professionals, insuring the healthcare inheritors and averting potential litigation and jurisdiction beyond local territories are of utmost consequence. Data protection and custody, fundamental data interchange, patient obscurity, using the morse alphabet must be decreed. Written authorization of the patient is desirable. Personal data is vital only when legislated. For cross border interrelation, a guarded and classified data protection needs to be metamorphosed to a national law. Data flow would only ensue where cross border agreement can be cultivated. For reimbursable services, qualitative and quantitative decisions can be made at the cross border and cross regional levels to curtail disruption of the health system. Governance propositions and alliances, to avert distractions encompass conjoint standards of care and indemnification. Legislation is necessitated for entitlement as well as accountability. A bilateral ratification is vital on the choice of law and designated court of judicature.

\section{Acknowledgements}

None.

\section{Conflict of interest}

The author declares no conflict of interest.

\section{References}

1. Giard RW. When is the practice of pathology malpractice. J Clin Pathol. 2010;63(11):957-961 